\title{
Do oxysterols control cholesterol homeostasis?
}

\author{
Ingemar Björkhem \\ Division of Clinical Chemistry, Karolinska Institutet, Huddinge University Hospital, Huddinge, Sweden \\ J. Clin. Invest. 110:725-730 (2002). doi:10.1172/JCI200216388.
}

Oxysterols are oxygenated derivatives of cholesterol with a very short half-life relative to cholesterol. As a consequence they are present in very low concentrations in all mammalian systems, almost invariably accompanied by $10^{3}$ - to $10^{6}$-fold excess of cholesterol. Oxysterols are important intermediates in a number of hepatic and extrahepatic catabolic pathways, most of which generate water-soluble bile acids as final products. Based on largely indirect evidence, and in spite of their low levels in vivo, oxysterols are generally believed to be important physiological mediators of cholesterol-induced effects. Perhaps the best support for this model is the existence of nuclear receptors that bind these compounds with high affinity and the fact that oxysterols potently regulate the expression of sterol-sensitive genes in vitro. Here I consider the role of oxysterols as intermediates in different catabolic pathways, and I weigh the evidence for and against the "oxysterol hypothesis" of cholesterol homeostasis.

\section{Oxygenation in cholesterol metabolism}

Cholesterol synthesis requires only one oxygenation reaction, but several such steps are necessary in its degradation to bile acids and its conversion to steroid hormones. Introduction of an oxygen atom in cholesterol drastically reduces its half-life and directs the molecule to leave the body. The physical properties of oxysterols facilitate their degradation and excretion, as oxysterols are able to pass lipophilic membranes much more quickly than does cholesterol itself (1).

While all cells may have at least some ability to oxygenate cholesterol, the liver and the endocrine organs have the highest capacity for such reactions. The liver is key in this regard, since it contains all of the enzymes required to convert an oxysterol into a water-soluble bile acid that can be excreted in bile. The most important oxygenases active on cholesterol all belong to the cytochrome P-450 family. Because of the irreversible

\footnotetext{
Address correspondence to: Ingemar Björkhem, Division of Clinical Chemistry, Karolinska Institutet, Huddinge University Hospital, SE-141 86 Huddinge, Sweden. Phone: 46-8-585-812-35; Fax: 46-8-585-812-60; E-mail: Ingemar.Bjorkhem@chemlab.hs.sll.se. Conflict of interest: No conflict of interest has been declared. Nonstandard abbreviations used: sterol-responsive element (SRE); sterol-responsive element-binding protein (SREBP).
}

catabolic consequences of the oxygenation, there is a need for metabolic control of the cytochrome P-450 species that act on cholesterol, particularly the rate-limiting oxygenation reactions associated with the major pathways for bile acid production.

\section{Hepatic oxysterols as intermediates in bile acid synthesis}

The major pathway in the biosynthesis of bile acids in mammalian liver starts with a hydroxylation of cholesterol in $7 \alpha$-position (Figure 1). This hydroxylation is rate-limiting in the overall conversion and is catalyzed by a microsomal cytochrome P-450 (CYP7A1), a well -studied enzyme whose transcriptional regulation may be somewhat different in dufferent species (2-5). For instance, the nuclear receptor LXR $\alpha$ is important for the regulation of the enzyme in rodents but not in humans. However, CYP7A1 is principally regulated under normal conditions by negative feedback regulation by bile acids reabsorbed from the intestine, which reach the liver via the portal vein.

In addition to the pathway initiated by CYP7A1, there is an alternative pathway starting with a hydroxylation of cholesterol in 27-position (Figure 1). This hydroxylation is catalyzed by a mitochondrial cytochrome P-450 (CYP27), an enzyme present in most tissues and organs. Because CYP27 can oxidize the terminal methyl group not only into a $\mathrm{CH}_{2} \mathrm{OH}$ group but also into a carboxylic acid (to form cholestenoic acid), this alternative route to bile acid synthesis is called the "acid" pathway. CYP27 also participates in the major pathway to bile acids by mediating the conversion of the $\mathrm{C}-27$ steroid side-chain into the C-24 steroid side-chain. As with CYP7A1, hepatic CYP27 may be regulated by a number of factors, including the enterohepatic flux of bile acids. Considerable species variation exists, however, and human hepatic CYP27 seems to be relatively insensitive to regulation at a transcriptional level (for a review, see ref. 5).

Another, more minor hepatic pathway converts 24S-hydroxycholesterol formed in the brain by CYP46 (Figure 1) into bile acids, but the details of this process are not clear. At least half of the $24 \mathrm{~S}$-hydroxycholesterol is excreted in bile in sulfated and/or glucuronidated form or as a corresponding conjugate of 24,27-dihydroxycholesterol (6) (Figure 2). 


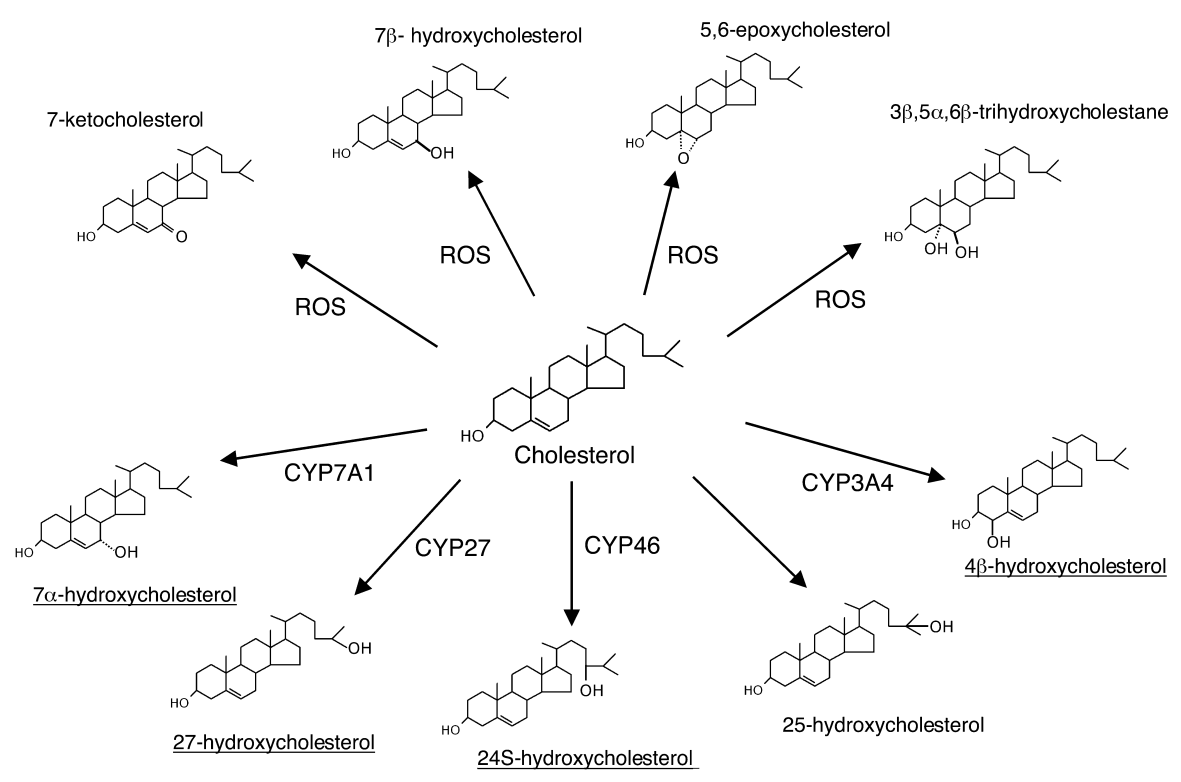

Figure 1

Primary cholesterol oxygenation reactions mediated by different cytochrome P-450 species or occurring nonenzymatically in the presence of reactive oxygen species (ROS) (43). The oxygenation of cholesterol into 25-hydroxycholesterol is catalyzed by cholesterol 25-hydroxylase, a non-heme iron protein (7). The quantitatively most important oxysterols present in human circulation are underlined.

25-Hydroxycholesterol (Figure 1) is a minor oxysterol that may be formed in different types of tissues by a specific enzyme not belonging to the cytochrome P-450 family (7). The mode of regulation of this enzyme has not been defined. 25-Hydroxycholesterol is known to be converted into normal bile acids in the liver, although less efficiently than other compounds, such as $7 \alpha$-hydroxycholesterol.

Very recently it was shown that one of the major oxysterols in the circulation, $4 \beta$-hydroxycholesterol (Figure 1), is formed from cholesterol in the liver and possibly also in the intestine by the cytochrome P-450 species CYP3A4 (8). While the metabolic end products of $4 \beta$-hydroxycholesterol have not yet been defined, the existence of $4 \beta$-hydroxylated bile acids in humans suggests that this oxysterol can be metabolized in the liver.

\section{Oxysterols as transport forms of cholesterol}

Due to the ability of oxysterols to pass cell membranes and to equilibrate with lipoproteins at a much faster rate than that of cholesterol, formation of an oxysterol is a mechanism by which some cells may eliminate excess cholesterol. The most important oxysterols involved in such transport mechanisms are side-chain oxidized oxysterols with an oxygen function in 24- or 27-position. After reaching the circulation, such side-chain oxidized oxysterols are rapidly taken up by the liver and further oxidized into bile acids or other water-soluble metabolites. This

\section{Figure 2}

Roles of CYP27 and CYP46 in transport of cholesterol from extrahepatic organs to the liver. Cholesterol does not pass readily across the blood-brain barrier, but cholesterol produced in the brain can be oxidized by CYP46 to form the more soluble species 24S-hydroxycholesterol. Likewise, 27-hydroxycholesterol is formed in other extrahepatic cells and contributes to the net flow of circulating oxysterols from other organs to the liver (10). mechanism can be regarded as an alternative to the classical reversed cholesterol transport mediated by ABC transporters and HDL (see Tall et al., this Perspective series, ref. 9).

Two quantitatively significant mechanisms for oxysterol elimination are known, one meditated by CYP27, and the other by CYP46 (Figure 2). 27-Oxygenated cholesterol metabolites flow continuously from peripheral tissues to the liver (10) and are thought to represent precursors for about $5-10 \%$ of total hepatic bile acid production $(10,11)$. Macrophages, which express high levels of CYP27, may depend on oxidative elimination of cholesterol by this pathway in order to avoid cholesterol accumulation. Indeed, cholesterol is found to accumulate in cultured human macrophages exposed to an inhibitor of CYP27 $(10,12)$. Consistent with the view that CYP27 is an antiatherogenic enzyme, patients

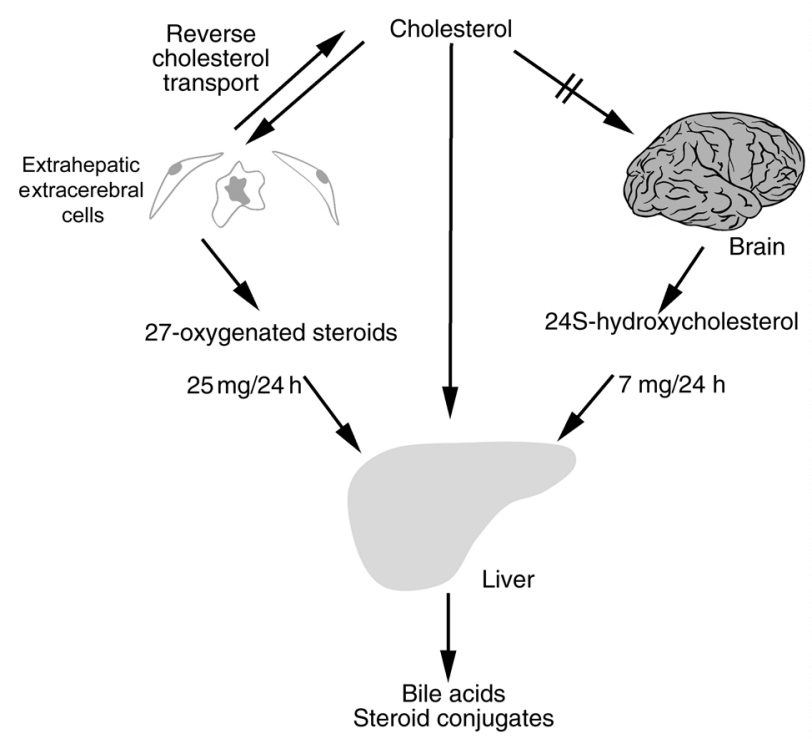


with cerebrotendinous xanthomatosis lack a normal copy of CYP27 and are at risk of premature atherosclerosis despite the normal levels of cholesterol in their circulation (13). Furthermore, as discussed below, mice with the corresponding null mutation do not develop atherosclerosis, at least not on a normal diet. In vitro, the sterol 27-hydroxylase pathway appears to be most important when cells are not provided with an extracellular acceptor such as HDL (10). Indeed, we (unpublished observations) have found some signs that the sterol 27-hydroxylase mechanism is modestly upregulated in Tangier disease, where HDL is deficient (see Tall et al., this Perspective series, ref. 9).

CNS neurons contain the CYP46 cytochrome P-450 species, which hydroxylates cholesterol to yield 24Shydroxycholesterol $(10,14)$. Unlike cholesterol, this oxysterol can pass the blood-brain barrier. In humans, the resulting flux of 24S-hydroxycholesterol from the brain into the circulation is continuous and is similar in magnitude to hepatic uptake of this species (Figure 2), indicating that most of the $24 \mathrm{~S}$-hydroxycholesterol present in the circulation originates from the brain. In rat brains, the rate of $24 \mathrm{~S}$-hydroxycholesterol synthesis is matched by the rate of synthesis of cholesterol, suggesting that there is a balance between synthesis and metabolism of cholesterol (15). The contribution of CYP46 to CNS cholesterol homeostasis in the human brain is not known, and cholesterol can also be transported from the brain into the cerebrospinal fluid by a mechanism mediated by apoE (16). Under normal conditions, this mechanism appears to be of little quantitative importance for efflux of cholesterol from the brain, but it may play a large part in transport within the human brain.

\section{Oxysterols in the circulation}

Due to the high levels of cholesterol in the circulation and the risk of autoxidation to yield 5,6- and 7-oxygenated oxysterols during isolation and workup, it has long been uncertain whether 7-ketocholesterol, $7 \beta$-hydroxycholesterol, 5,6-epoxycholesterol, and $3 \beta, 5 \alpha, 6 \beta$-trihydroxycholestane exist in vivo (Figure 1). To address this question, we (17) exposed rats to ${ }^{18} \mathrm{O}_{2}$ and analyzed the resulting ${ }^{18} \mathrm{O}$-labeled oxysterols in the animals' plasma. We observed significant incorporation of ${ }^{18} \mathrm{O}$ into all oxysterols except the 5,6-oxygenated steroids, which we therefore regard as probable methodological artifacts. Indeed, these oxysterols generally seem to disappear when extraction and chromatography are conducted in an oxygen-free environment. Conversely, the other species appear to be formed in vivo by oxygendependent oxygenases or by direct attack of active oxygen species in nonenzymatic reactions.

Most of the $7 \alpha$-hydroxycholesterol present in the circulation is probably produced by hepatic CYP7A1, since the activity of this enzyme covaries with plasma levels of $7 \alpha$-hydroxycholesterol (18). On the other hand, most of the $7 \beta$-hydroxycholesterol and 7-ketocholesterol present in the circulation may be secondary products of lipid peroxidation. Interestingly, the levels of $7 \beta$-hydroxycholesterol appear to be increased in connection with cardiovascular disease (19). Circulating 27-hydroxycholesterol, which is mainly of extrahepatic origin, is likewise found at high levels in some patients with advanced atherosclerosis, but this is not a general finding.

Plasma levels of 24S-hydroxycholesterol have been shown to reflect the balance between cerebral production and hepatic metabolism of the oxysterol (20) and are highest during the first years of life, decreasing by a factor of about four during the first two decades of life. During this period the ratio between the size of the brain and the size of the liver decreases by a factor of about three. The levels of 24S-hydroxycholesterol may also decrease as a consequence of severe neurological diseases affecting the number of neurons in the brain (21).

Plasma levels of $4 \beta$-hydroxycholesterol are likely to reflect the amount of CYP3A4 present in the liver. Patients treated with antiepileptics and other drugs that upregulate this cytochrome may have levels of $4 \beta$-hydroxycholesterol 10- to 20-fold higher than normal (8).

Cholesterol is currently thought to exist in vivo in three distinct pools, distinguished by their rates of exchange with plasma cholesterol: One pool exchanges rapidly, one slowly, and one essentially not at all. Recent studies in which deuterium-labeled cholesterol were infused at high levels into a human volunteer showed that $7 \alpha$-hydroxycholesterol in the human circulation originates from the pool that is in rapid equilibration with plasma cholesterol (22). Most of 27-hydroxycholesterol originates from the pool that is only slowly exchangeable. In accordance with the contention that most or all 24-hydroxycholesterol originates from brain cholesterol, this oxysterol reflects the pool with practically no exchange with plasma cholesterol. Thus, each of the three major oxysterols in the circulation can be regarded as a marker for a specific pool of cholesterol in the body.

\section{Oxysterols as potential regulators of cholesterol homeostasis}

Cholesterol- and oxysterol-induced transcriptional silencing has been defined in detail (23) and is known to involve a sterol-responsive element (SRE) in the promoter of target genes. This sequence is recognized by a sterol-responsive element-binding protein (SREBP), which initiates transcription. As recently reviewed by Horton et al. in the JCI (23), the aminoterminal half of an SREBP precursor is cleaved from the membranes by a series of proteolytic steps in cells depleted of cholesterol. The peptide released has a DNA-binding domain that binds to the SRE sequence and a transactivation domain that modulates the transcription activity. In the presence of sterols - either cholesterol or oxysterols - no such proteolytic cleavage occurs. A specific protein, SREBP cleavage-activating protein (SCAP), is responsible for the regulation of the cleavage of SREBP and may be 
regarded as a sensor for the levels of the steroids in the cell. Although cholesterol synthesis in isolated cultured cells can be suppressed by either exogenous cholesterol or oxygenated cholesterol derivatives, the concept that the short-lived intermediates in cholesterol catabolic pathways, rather than cholesterol itself, downregulate cholesterol synthesis is very attractive. In particular, 25-hydroxycholesterol has been found to be one of the most potent suppressors of sterol synthesis in cultured cells, and this specific oxysterol has been used as a general tool to identify sterol-sensitive genes.

Many but by no means all of the available data are consistent with this oxysterol hypothesis. Initial support for this idea came from the work of Kandutsch et al. (24), who found that oxysterols are much more active than cholesterol itself in suppressing cholesterol synthesis; indeed, oxysterols are potent suppressors at concentrations as low as $10^{-9} \mathrm{M}$, whereas cholesterol is only weakly inhibitory at $10^{-5} \mathrm{M}$. Also consistent with the idea that contaminating oxysterols account for the transcriptional response, the inhibitory effect of cholesterol was found to disappear after addition of vitamin $\mathrm{E}$ to the medium. Following up on this experiment, Kandutsch and Thompson characterized a cytosolic protein whose affinities for different oxysterols correlated with the potency of the oxysterol to suppress HMG CoA reductase in fibroblasts (25). Addition of 25-hydroxycholesterol causes it to redistribute within the cell and become associated with the Golgi apparatus, suggesting a role for this protein in the intracellular transport of oxysterols. This protein, now known to be one of a family of oxysterol-binding proteins (26), seems to be present in most or all animal cells.

In vivo studies by Saucier et al. (27) have shown that several oxysterols accumulate in mouse liver after cholesterol feeding, suggesting that these oxysterols could act through one or more of the oxysterol-binding proteins to downregulate HMG CoA reductase and block endogenous cholesterol synthesis. The levels of the accumulating oxysterols found were sufficient to explain the observed downregulation, as judged by data obtained from in vitro studies with pure oxysterols. While these data are consistent with the oxysterol hypothesis, the concern remains that the physiological ligands of these proteins are not known with certainty, particularly since some of the members of the oxysterol-binding family bind phospholipids and may be of importance for vesicular transport. Moreover, the addition of pure oxysterols to in vitro systems can be regarded as highly nonphysiological.

While such experiments can give useful information concerning the sterol-sensitivity of different genes, no firm conclusions can be drawn concerning oxysterols' normal functions, since oxysterols in vivo are always present together with $10^{3}$ - to $10^{6}$-fold excess of cholesterol. It has been shown that some biological effects of oxysterols are markedly reduced even when they are moderately diluted with cholesterol (28). Furthermore, while the oxysterol-binding nuclear receptors exhibit a very high affinity for oxysterols under in vitro conditions, with little or no measurable affinity for cholesterol, this specificity has not been tested in a physiological range. Hence, it remains to be established whether these nuclear receptors can interact specifically with oxysterols that are present in a mixture containing a more than $10^{4}$-fold excess of cholesterol.

Another argument in favor of the oxysterol hypothesis derives from experiments with cultured cells, indicating that blockade of the cytochrome P-450 system prevents the normal LDL-induced suppression of cholesterol synthesis (for a review, see ref. 29). The specificity of the cytochrome P-450 inhibitors used in these experiments may, however, be questioned, and it has not been possible to link these effects to a specific oxysterol.

Finally, the recent identification of nuclear receptors LXR $\alpha$ and LXR $\beta$ as regulators of cholesterol metabolism has been taken as strong evidence that oxysterols mediate cellular cholesterol homeostasis. LXR $\alpha$ strongly modulates the transcription of the cholesterol transporters $\mathrm{ABCA} 1$ and $\mathrm{ABCG} 1$, which participate in cholesterol flux from enterocytes and macrophages, respectively (for a review, see ref. 30). The LXRs also seem to regulate human cholesteryl ester transfer protein, and LXR $\alpha$ is an important regulator of cholesterol $7 \alpha$-hydroxylase in mice (4). LXR $\alpha$ and LXR $\beta$ bind with high affinity to oxysterols, especially 24S-hydroxycholesterol and 24,25-epoxycholesterol, and oxysterols are generally believed to be the most important physiological activators for these receptors. However, whether or not the oxysterols are ligands for these receptors under in vivo conditions is not known with certainty, and it is difficult to exclude a role for unmodified cholesterol, as either a ligand for or an antagonist to these receptors.

In addition to the various caveats that plague some of the arguments favoring the oxysterol hypothesis, there is also a body of data that seem difficult to square with this idea. For instance, while administration of dietary cholesterol to mice leads to a marked suppression of cholesterol synthesis in the liver, replacement of cholesterol with different derivatives that reduce or block hydroxylation of positions 7,24 , and 27 fails to prevent the suppressive effect of the sterol on hepatic cholesterol synthesis (for a review, see ref. 29). Erickson and Nes have shown that even a complete elimination of the steroid side-chain of cholesterol does not prevent the inhibitory effect on hepatic cholesterol downregulation in this mouse model (31). Likewise, recent experiments with genetically engineered mice have shown that even marked changes in plasma levels of specific oxysterols (25-hydroxycholesterol and 27-hydroxycholesterol) lead to surprisingly small changes in cholesterol turnover and homeostasis $(32,33)$.

The problems in evaluating the literature on this subject may be illustrated by one specific oxysterol, 27-hydroxycholesterol - one of the major oxysterols in the circulation. In view of its strong suppression of cholesterol synthesis in cultured cells and its continuous flux from peripheral sources to the liver, 
27-hydroxycholesterol would appear a very attractive candidate to be a regulatory steroid. However, the data from genetically engineered mice and mice fed cholesterol derivatives that cannot be modified at the 27 position weaken this hypothesis substantially. The fact that humans lacking CYP27, the enzyme that generates 27 -hydroxycholesterol, have normal circulating levels of cholesterol also argues that this oxysterol is not uniquely required for cholesterol homeostasis. One report on CYP27-deficient cultured human fibroblasts (34) suggests that formation of 27-hydroxycholesterol may be important for the normal suppression of cholesterol synthesis by LDL, but another group has reached a different conclusion (35). In a very recent work, Fu et al. (36) found evidence that cholesterol 27-hydroxylation can activate LXR, resulting in increased activity of the cholesterol transporters ABCA1 and ABCG1 in cholesterol-loaded skin fibroblasts and monocyte-derived macrophages. In two other studies, however, 27-hydroxycholesterol was not found to be an efficient activator of human LXR (37, 38 ). Based on an in vitro study on levels of oxysterols in different subcellular fractions of the livers of mice exposed to dietary cholesterol, Zhang et al. recently concluded that 27-hydroxycholesterol is not likely to be an important regulator of cholesterol homeostasis, at least not at a transcriptional level (39), since this steroid fails to accumulate in the nucleus after administration of cholesterol. Thus, most studies to date do not support the contention that 27-hydroxycholesterol represents an important regulator of cholesterol homeostasis.

\section{Oxysterols and atherosclerosis}

When added to cultured endothelial cells and arterial smooth muscle cells, oxysterols are cytotoxic and may also induce apoptosis (see Tabas, this Perspective series, ref. 40). Based on such experiments, oxysterols have been suggested to be atherogenic, but the highly nonphysiological conditions used in most experiments preclude strong conclusions. Since oxysterols mimic many of the effects of cholesterol, dietary oxysterols might be expected to be atherogenic, but clear evidence for this is lacking. A great number of studies have been published on effects of dietary oxysterols on experimental animals (for an excellent review, see ref. 41). Of 13 such studies, six indicate a proatherogenic effect, four an antiatherogenic effect, while three show no significant effect.

Oxidatively modified LDL, whose proatherogenic effect is usually explained by its internalization via unregulated scavenger receptors, contains 7-hydroperoxycholesterol and its more stable degradation products $7 \alpha$ - and $7 \beta$-hydroxycholesterol and 7 -ketocholesterol. 7-Hydroperoxycholesterol appears to be the most cytotoxic oxygenated lipid in oxidized LDL (42) and may well be a powerful atherogenic factor. Unfortunately, studies to address this possibility are difficult because of the compound's great lability. Toxic oxysterols associated with modified lipoproteins may thus represent an additional atherogenic factor, over and above the simple accumulation of cholesterol in foam cells in the developing atherosclerotic lesion. If so, it will be important to consider the role of other oxysterols in modifying this risk. In particular, since the putative antiatherogenic enzyme CYP27 appears to be highly active in human atheromas, it may be that one oxysterol, 27-hydroxycholesterol, participates in a defense mechanism that suppresses cholesterol accumulation in the presence of 7-hydroperoxycholesterol or other harmful lipids.

\section{Acknowledgments}

The studies referred to from the author's laboratory have been supported by grants from the Swedish Medical Research Council and the Swedish HeartLung Foundation.

1. Lange, Y., Ye, J., and Strebel, F. 1995. Movement of 25-hydroxycholesterol from the plasma membrane to the rough endoplasmic reticulum in cultured hepatoma cells. J. Lipid Res. 36:1092-1097.

2. Russell, D.W. 1999. Nuclear orphan receptors control cholesterol catabolism. Cell. 97:539-542.

3. Repa, J.J., and Mangelsdorf, D.J. 2000. The role of orphan nuclear receptors in the regulation of cholesterol homeostasis. Annu. Rev. Cell Dev. Biol. 16:459-481.

4. Chiang, J.Y.L., Kimmel, R., and Stroup, D. 2001. Regulation of cholesterol $7 \alpha$-hydroxylase gene (CYP7A1) transcription by the liver orphan receptor (LXR $\alpha)$. Gene. 262:257-265.

5. Björkhem, I., and Eggertsen, G. 2001.Genes involved in initial steps of bile acid synthesis. Curr. Opin. Lipidol. 12:97-103.

6. Björkhem, I., et al. 2001. From brain to bile. Evidence that conjugation and $\omega$-hydroxylation are important for elimination of 24 S-hydroxycholesterol (cerebrosterol) in humans. J. Biol. Chem. 276:37004-37010.

7. Lund, E.G., Kerr, T.A., Sakai, J., Li, W.-P., and Russell, D.W. 1998. cDNA cloning of mouse and human cholesterol 25-hydroxylases, polytopic membrane proteins that synthesize a potent oxysterol regulator of lipid metabolism. J. Biol. Chem. 273:34316-34327.

8. Bodin, K., et al. 2001. Antiepileptic drugs increase plasma levels of $4 \beta$-hydroxycholesterol in humans. Evidence for involvement of cytochrome P450 3A4. J. Biol. Chem. 276:38685-38689.

9. Tall, A.R., Costet, P., and Wang, N. 2002. Regulation and mechanisms of macrophage cholesterol efflux. J. Clin. Invest. In press. doi:10.1172/JCI200216391.

10. Björkhem, I., Diczfalusy, U., and Lütjohann, D. 1999. Removal of cholesterol from extrahepatic sources by oxidative mechanisms. Curr. Opin. Lipidol. 10:161-165.

11. Duane, W.C., and Javitt, N.B. 1999. 27-Hydroxycholesterol: production rates in normal human subjects. J. Lipid Res. 40:1194-1199.

12. Lund, E.G., Andersson, O., Zhang, J., et al. 1996. Importance of a novel oxidative mechanism for elimination of intracellular cholesterol in humans. Arterioscler. Thromb. Vasc. Biol. 16:208-212.

13. Björkhem, I., Muri Boberg, K., and Leitersdorf, E. 2001. Inborn errors in bile acid biosynthesis and storage of sterols other than cholesterol. In The metabolic bases of inherited disease. C.R. Scriver et al., editors. McGraw-Hill. New York, New York, USA. 2961-2988.

14. Lund, E.G., Guileyardo, J.M., and Russell, D.W. 1999. cDNA cloning of cholesterol 24-hydroxylase, a mediator of cholesterol homeostasis in the brain. Proc. Natl. Acad. Sci. USA. 96:7238-7243.

15. Björkhem, I., Lütjohann, D., Breuer, O., Sakinis, A., and Wennmalm, Å. 1997. Importance of a novel oxidative mechanism for elimination of brain cholesterol. J. Biol. Chem. 272:30178-30184.

16. Pitas, R.E., Boyles, J.K., Lee, S.H., Foss, D., and Mahley, R.W. 1987. Astrocytes synthesize apolipoprotein $\mathrm{E}$ and metabolize apolipoprotein E-containing lipoproteins. Biochim. Biophys. Acta. 917:148-161.

17. Breuer, O., and Björkhem, I. 1995. Use of an ${ }^{18} \mathrm{O}_{2}$ inhalation technique and mass isotopomer distribution analysis to study oxygenation of cholesterol in rat. Evidence for in vivo formation of 7-oxo-, $7 \beta$ hydroxy-, 24-hydroxy-, and 25-hydroxycholesterol. J. Biol. Chem. 270:20278-20284.

18. Björkhem, I., et al. 1987. On the possible use of the serum level of $7 \alpha$-hydroxycholesterol as a marker for increased activity of the cholesterol $7 \alpha$-hydroxylase. J. Lipid Res. 28:889-894.

19. Salonen, J.T., et al. 1997. Lipoprotein oxidation and progression of carotid atherosclerosis. Circulation. 95:840-845.

20. Bretillon, L., Lütjohann, D., Ståhle, L., et al. 2000. Plasma levels of 24S-hydroxy-cholesterol reflect the balance between cerebral production and hepatic metabolism and are inversely related to body surface. J. Lipid Res. 41:840-845 
21. Bretillon, L., Sidén, Å., Wahlund, L.-O., et al. 2000. Plasma levels of 24S-hydroxy-cholesterol in patients with neurological diseases. Newrosci. Lett. 293:87-90.

22. Meaney, S., et al. 2001. Evidence that the major oxysterols in human circulation originate from distinct pools of cholesterol: a stable isotope study. J. Lipid Res. 42:70-78.

23. Horton, J.D., Goldstein, J.L., and Brown, M.S. 2002. SREBPs: activators of the complete program of cholesterol and fatty acid synthesis in the liver. J. Clin. Invest. 109:1125-1131. doi:10.1172/JCI200215593.

24. Kandutsch, A.A., Chen, H.W., and Heiniger, H.-J. 1978. Biological activity of some oxygenated sterols. Science. 201:498-501.

25. Kandutsch, A.A., and Thompson, E.B. 1980. Cytosolic proteins that bind oxygenated sterols. J. Biol. Chem. 255:10813-10826.

26. Xu, Y.Y., Liu, X., Ridgway, N.D., and McMaster, C.R. 2001. Novel members of the human oxysterol-binding protein family bind phospholipids and regulate vesicle transport. J. Biol. Chem. 276:18407-18414

27. Saucier, S.E., Kandutsch, A.A., Gayen, A.K., Swahn, D.K., and Spencer, T.A. 1989. Oxysterol regulators of 3-hydroxy-3-methylglutaryl-CoA reductase in liver. J. Biol. Chem. 264:6863-6869.

28. Clare, K., Hardwick, S.J., Carpenter, K.L.H., Weeratunge, N., and Mitchinson, M.J. 1995. Toxicity of oxysterols to human monocytemacrophages. Atherosclerosis. 118:67-75.

29. Lund, E.G., and Björkhem, I. 1995. Role of oxysterols in the regulation of cholesterol homeostasis: a critical evaluation. Acc. Chem. Res. 28:241-249.

30. Chawla, A., Repa, J.J., Evans, R.M., and Mangelsdorf, D.J. 2001. Nuclear receptors and lipid physiology: opening the X-files. Science. 294: $1866-1870$.

31. Erickson, K.A., and Nes, W.R. 1982. Inhibition of hepatic cholesterol synthesis in mice by sterols with shortened and stereochemically varied side chains. Proc. Natl. Acad. Sci. USA. 79:4873-4877.

32. Rosen, H., et al. 1998. Markedly reduced bile acid synthesis but maintained levels of cholesterol and vitamin D metabolites in mice with disrupted sterol 27-hydroxylase gene. J. Biol. Chem. 273:14805-14812.

33. Li-Hawkins, J., Lund, E.G., Turley, S.D., and Russell, D.W. 2000. Disruption of the oxysterol $7 \alpha$-hydroxylase gene in mice. J. Biol. Chem 275:16536-16542.

34. Axelson, M., and Larsson, O. 1995. Low density lipoprotein (LDL) cholesterol is converted to 27 -hydroxycholesterol in human fibroblasts. J. Biol. Chem. 270:15102-15110.

35. Tint, G.S., and Salen G. 1982. Biosynthesis of cholesterol, lanosterol, and $\Delta^{7}$-cholestenol, but not cholestanol, in cultured fibroblasts from normal individuals and patients with cerebrotendinous xanthomatosis. J. Lipid Res. 23:597-603.

36. Fu, X., et al. 2001. 27-Hydroxycholesterol is an endogenous ligand for liver X receptor in cholesterol-loaded cells. J. Biol. Chem. 42:38378-38387.

37. Lehmann, J.M., et al. 1997. Activation of the nuclear receptor LXR by oxysterols defines a new hormone response pathway. J. Biol. Chem. 272:3137-3140.

38. Janowski, B.A., Willy, P.J., Devi, T.R., Falck, J.R., and Mangelsdorf, D.J. 1996. An oxysterol signalling pathway mediated by the nuclear receptor LXR $\alpha$. Nature. 383:728-731.

39. Zhang, Z., et al. 2001. Key regulatory oxysterols in liver: analysis as $\Delta^{4}$-3ketone derivatives by HPLC and response to physiological perturbations. J. Lipid Res. 42:649-658.

40. Tabas, I. 2002. Consequences of cellular cholesterol accumulation: basic concepts and physiological implications. J. Clin. Invest. In press. doi:10.1172/JCI200216452.

41. Brown, A.J., and Jessup, W. 1998. Oxysterols and atherosclerosis. Atherosclerosis. 142:1-28.

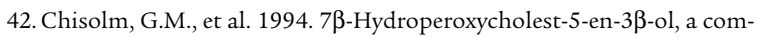
ponent of human atherosclerotic lesions, is the primary cytotoxin of oxidized human low density lipoprotein. Proc. Natl. Acad. Sci. USA. 91:11452-11456.

43. Smith, L.L. 1981. Cholesterol autoxidation. Plenum Press. New York, New York, USA. 1-674. 